GLOBAL JOURNAL OF PURE AND APPLIED SCIENCES VOL. 27, 2021: 43-53 COPYRIGHT@ BACHUDO SCIENCE CO. LTD PRINTED IN NIGERIA ISSN 1118-0579

\title{
A NEW WEIBULL EXPONENTIATED INVERTED WEIBULL DISTRIBUTION FOR MODELLING POSITIVELY-SKEWED DATA
}

\author{
BRAIMAH J. O., ADJEKUKOR J. A., EDIKE N. AND ELAKHE S. O.
}

(Received 12 November 2020; Revision Accepted 27 November 2020)

\begin{abstract}
An Exponentiated Inverted Weibull Distribution (EIWD) has a hazard rate (failure rate) function that is unimodal, thus making it less efficient for modeling data with an increasing failure rate (IFR). Hence, the need to generalize the EIWD in order to obtain a distribution that will be proficient in modeling these types of dataset (data with an increasing failure rate). This paper therefore, extends the EIWD in order to obtain Weibull Exponentiated Inverted Weibull (WEIW) distribution using the Weibull-Generator technique. Some of the properties investigated include the mean, variance, median, moments, quantile and moment generating functions. The explicit expressions were derived for the order statistics and hazard/failure rate function. The estimation of parameters was derived using the maximum likelihood method. The developed model was applied to a real-life dataset and compared with some existing competing lifetime distributions. The result revealed that the (WEIW) distribution provided a better fit to the real life dataset than the existing Weibull/Exponential family distributions.
\end{abstract}

KEYWORDS Hazard function, Log-likelihood, Failure rate, Moment generating function, Order statistics

\section{INTRODUCTION}

Generalization of a probability distribution is a common technique used for introducing flexibility to classical distributions through the introduction of parameters or reduction of some identified redundant parameters. According to Alzaatreh, Lee and Famoye, the Weibull distribution was named after Walodi Weibull and has been used to model some families of distributions by several authors with the intention of generating more flexible distributions [1]. The Weibull- $X$ family was proposed by [1] while Bourguignon, Silva and Cordeiro proposed the Weibull-G family [2]. Several flexible distributions have been developed from these important families of distributions. Alzaatreh, Famoye and Lee developed Weibull-Pareto [3] while Aljarrah, Famoye and Lee developed a new Weibull-Pareto distribution [4]. Merovci and Elbatal developed Weibull Rayleigh distribution [5]. Oguntunde, Balogun, Okagbue and Bishop developed the Weibull Exponential distribution [6] while Yahaya and Sa'ad introduced the Weibull-Burr XII distribution [7].
By means of the inverse transformation of variables, some baseline distributions have been inverted and generalized. Some of the existing results revealed that inverted distributions when extended can also provide a more flexible distribution than the baseline distribution. Voda introduced the use of Inverse Rayleigh (IR) distribution [8] while the Inverse Exponential (IE) and their study revealed that the IW is useful for modeling systems with failure rates common in biological and reliability studies. Drapella and Mudholkar and Kolia as well studied the Inverse Weibull Distribution and projected complementary Weibull and reciprocal Weibull as another names for the model [9], [10] while Khan, Pasha, and Pasha studied the flexibility of IW distribution [11]. De Gusmao, Ortega, and Cordeiro proposed the Generalized Inverse Weibull (GIW) distribution [12] while Elbatal and Muhammed later developed the Exponentiated Generalized Inverse Weibull (EGIW) Distribution [13].

It was also observed that generalization of distribution using the inverse of baseline distributions continues to achieve more attention among researchers in recent

Braimah J. O., Department of Mathematics and Statistics, P.M.B 14,Ambrose Alli University,Ekpoma, Edo State, Nigeria Adjekukor J. A., Department of Statistics, Delta State Polytechnic, Otefe-Oghara, Delta State, Nigeria Edike N., Department of Mathematics and Statistics, P.M.B 14,Ambrose Alli University,Ekpoma, Edo State, Nigeria

Elakhe S. O., Department of Mathematics and Statistics, P.M.B 14,Ambrose Alli University,Ekpoma, Edo State, Nigeria 
times as evident in the following studies.

Fatima, and Ahmad proposed the Weighted inverse Rayleigh distribution [14] while Oguntunde, Adejumo and Owoloko proposed the Weibull Inverted Exponential distribution [15]. The odd Frechet Inverse Weibull distribution was developed by [16], odd Frechet Inverse Rayleigh by [17], Inverse Weibull Inverse Exponential (IW-IE) distribution by [18], while [19] extended Inverse Rayleigh using the Half-Logistics transformation.

The IW is a useful model that attracts the attention of several researchers, it has been studied by several authors and also extended as Exponentiated Inverted Weibull (EIW) by [20]; the authors introduced the standard two-parameter exponentiated Inverted Weibull (EIW) distribution. [21] studied (EIW) distribution by investigating methods of parameter estimations using classical likelihood and Bayesian estimators for samples from complete and Type II censoring scheme. [22] extended (EIW) distribution by carrying out a comparative study of some estimation methods some of which are the MLE, least square, least line based on grouped data.
However, [23] in their investigation revealed that the EIW by [24] has a particular shortcoming, [23] proved that the standard two-parameter EIW has a unimodal shape that makes it ineffective in modeling data with increasing failure rate (IFR).Further extension on EIW was carried out by [25] using Quadratic Rank Transmutation Map (QRTM) and the Proposed Transmuted Exponentiated Inverse Weibull (TEIW) distribution applied to medical sciences data set.

This study is aimed at extending (EIW); the need for the study is motivated by the fact that preliminary findings revealed situations where the EIW model failed to provide a better fit than the IW model for some types of dataset. In [23], their findings also revealed that EIW has a hazard/failure rate function that is unimodal shape, thereby making it less effective for modeling data with increasing failure rate; hence it is necessary to generalize the EIW model to obtain a model that is capable of modeling such types of dataset. This study is using the Weibull Generalized class of distribution proposed by [2] to generalize the EIW.

\section{THEORY / CALCULATIONS}

\section{THE WEIBULL DISTRIBUTION}

The cumulative distribution function (cdf) of the Generalized Weibull family distribution is defined as:

$F(x)=\int_{0}^{\frac{G(x)}{1-G(x)}} \alpha \beta t^{\beta-1} e^{-\alpha t^{\beta}} d t$

$F(x)=1-e^{-\alpha\left[\frac{G(x)}{1-G(x)}\right]^{\beta}}$

while the corresponding density function is given as:

$f(x)=\alpha \beta g(x) \frac{[G(x)]^{\beta-1}}{[1-G(x)]^{\beta+1}} e^{-\alpha\left[\frac{G(x)}{1-G(x)}\right]^{\beta}}$

where $x>0, \alpha>0, \beta>0$

The Inverted Weibull (IW) distribution as proposed by [26] has the cdf and pdf given respectively as follows:

$F(x)=e^{-\left(\frac{\theta}{x}\right)^{k}}$

$f(x)=k \theta^{k} x^{-k-1} e^{-\left(\frac{\theta}{x}\right)^{k}}$

where $x>0, \theta>0, k>0 ; \theta$ is the quality or scale parameter while $k$ is the shape parameter.

The standard two-parameter Exponentiated Inverted Weibull (EIW) distribution was also proposed by [20] with the cdf and pdf defined respectively as:

$F(x ; \theta, \beta)=\left(e^{-x^{-\beta}}\right)^{\theta} ; x>0, \theta>0, \beta>0$

$f(x ; \theta, \beta)=\theta \beta x^{-(\beta+1)}\left(e^{-x^{-\beta}}\right)^{\theta}$

$x>0, \theta>0, \beta>2.22$

THE WEIBULL EXPONENTIATED INVERTED WEIBULL (WEIW) DISTRIBUTION

From equation (4), the cdf of Exponentiated Inverted Weibull (EIW) distribution is defined for this study as follow:

$G(x ; \theta, k, v)=\left(e^{-\left(\frac{\theta}{x}\right)^{k}}\right)^{v}$

The density function obtained from the derivative of equation (8) with respect to $x$ is given as:

$g(x)=b k \theta^{k} x^{-(k+1)} e^{-b\left(\frac{\theta}{x}\right)^{k}}$

$$
g(x)=v k \theta^{k} x^{-(k+1)}\left(e^{-\left(\frac{\theta}{x}\right)^{k}}\right)^{v}
$$

The proposed (WEIW) distribution has the cdf derived, using equations (2) and (8) as follow:

$F(x)=1-\exp \left(-\alpha\left[\frac{\left(e^{-\left(\frac{\theta}{x}\right)^{k}}\right)^{v}}{1-\left(e^{-\left(\frac{\theta}{x}\right)^{k}}\right)^{v}}\right]^{\beta}\right)$ 
The associated pdf of WEIW is obtained from the first derivative of $F(x)$ in equation (10) and is given as:

$$
\begin{aligned}
& f(x)=\alpha v \beta k \theta^{k} x^{-(k+1)} \frac{\left[\left(e^{-\left(\frac{\theta}{x}\right)^{k}}\right)^{v}\right]^{\beta}}{\left[1-\left(e^{-\left(\frac{\theta}{x}\right)^{k}}\right)^{v}\right]^{\beta+1}} \exp \left(-\alpha\left[\frac{\left(e^{-\left(\frac{\theta}{x}\right)^{k}}\right)^{v}}{1-\left(e^{-\left(\frac{\theta}{x}\right)^{k}}\right)^{v}}\right]^{\beta}\right) \\
& f(x)=\alpha \beta v k \theta^{k} x^{-(k+1)} \frac{\left[e^{-v\left(\frac{\theta}{x}\right)^{k}}\right]^{\beta}}{\left[1-e^{-v\left(\frac{\theta}{x}\right)^{k}}\right]^{\beta+1}} \exp \left(-\alpha\left[\frac{e^{-v\left(\frac{\theta}{x}\right)^{k}}}{1-e^{-v\left(\frac{\theta}{x}\right)^{k}}}\right]^{\beta}\right)
\end{aligned}
$$

where $x>0 ; \alpha, \beta>0$ and $b, k, \theta>0$.

The WEIW distribution has $\theta$ as scale parameter with $\alpha, \beta, v$ and $k$ as shape parameters
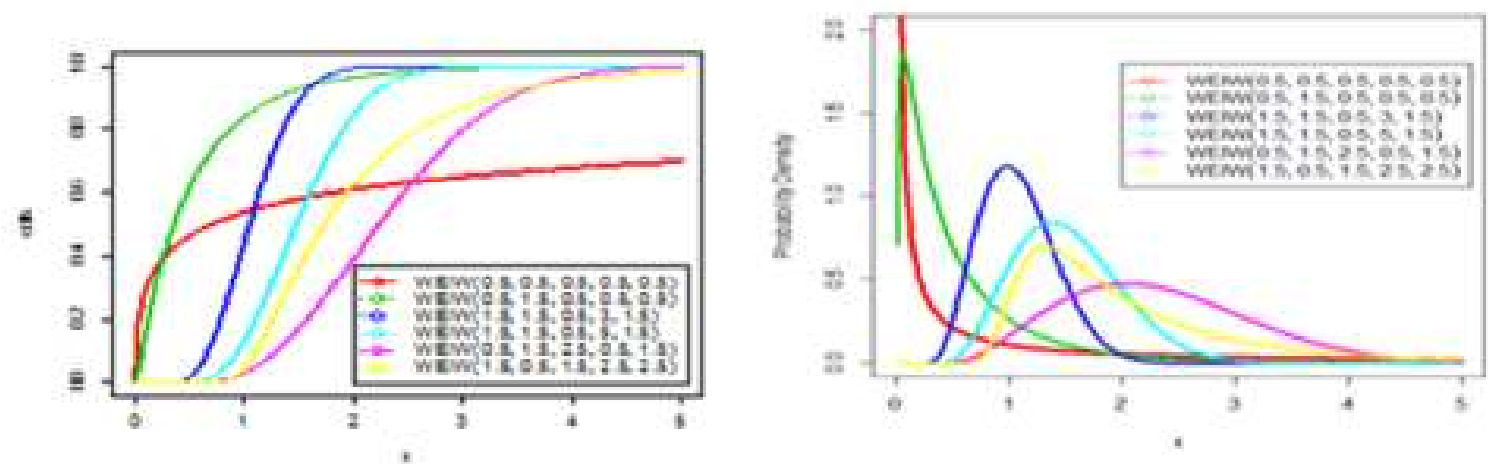

Figure 1: Plots of the cumulative distribution function and the probability density function

\section{DEVELOPMENT OF THE PROBABILITY FUNCTION OF WEIW DISTRIBUTION}

The probability density function of WIEW is written as:

$f(x)=\alpha \beta v k \theta^{k} x^{-k-1} \frac{\left[e^{-v\left(\frac{\theta}{x}\right)^{k}}\right]^{\beta}}{\left[1-e^{-v\left(\frac{\theta}{x}\right)^{k}}\right]^{\beta+1}} R(x)$

where $\mathrm{R}(\mathrm{x})=$ reliability function, $x>0 ; \alpha, \beta>0$ and $v, k, \theta>0$

On applying the power series expansion on the reliability function, the resulted function became:

$R(x)=\exp \left(-\alpha\left[\frac{e^{-v\left(\frac{\theta}{x}\right)^{k}}}{1-e^{-v\left(\frac{\theta}{x}\right)^{k}}}\right]^{\beta}\right)=\sum_{i=0}^{\infty}\left(\frac{(-1)^{i}}{i !}--\alpha^{i}\left[\frac{e^{-v\left(\frac{\theta}{x}\right)^{k}}}{1-e^{-v\left(\frac{\theta}{x}\right)^{k}}}\right]^{\beta i}\right)$

On substituting $R(x)$ into $f(x)$ and performing some arithmetic operations, we obtain:

$f(x)=\alpha v \beta k \theta^{k} x^{-k-1} \frac{\left[e^{-v\left(\frac{\theta}{x}\right)^{k}}\right]^{\beta}}{\left[1-e^{-v\left(\frac{\theta}{x}\right)^{k}}\right]^{\beta+1}} \sum_{i=0}^{\infty}\left(\frac{(-1)^{i}}{i !} \alpha^{i}\left[\frac{e^{-v\left(\frac{\theta}{x}\right)^{k}}}{\left.1-e^{-v\left(\frac{\theta}{x}\right)^{k}}\right]^{\beta i}}\right)\right.$
$f(x)=v \beta k \theta^{k} x^{-k-1} \sum_{i=0}^{\infty}\left(\frac{(-1)^{i}}{i !} \alpha^{i+1} \frac{\left[e^{-v\left(\frac{\theta}{x}\right)^{k}}\right]^{\beta(i+1)}}{\left[1-e^{-v\left(\frac{\theta}{x}\right)^{k}}\right]^{\beta(i+1)+1}}\right)$

Applying the binomial series expansion on equation (13) in the form:

$[1-Z]^{-a}=\sum_{j=0}^{\infty} \frac{(-1)^{j} \Gamma(a+j)}{j ! \Gamma(a)} Z^{j}$

$\left[1-e^{-v\left(\frac{\theta}{x}\right)^{k}}\right]^{-\beta(i+1)+1}=\sum_{j=0}^{\infty} \frac{(-1)^{j} \Gamma(\beta(i+1)+1+j)}{j ! \Gamma(\beta(i+1)+1)} e^{-v j\left(\frac{\theta}{x}\right)^{k}}$ 
On substituting equation (14) into equation (13), the resulting equations become:

$$
\begin{aligned}
& f(x)=v \beta k \theta^{k} x^{-k-1} \sum_{i=0}^{\infty} \frac{(-1)^{i}}{i !} \alpha^{i+1}\left[e^{-v\left(\frac{\theta}{x}\right)^{k}}\right]^{\beta(i+1)} \sum_{j=0}^{\infty} \frac{(-1)^{j} \Gamma(\beta(i+1)+1+j)}{j ! \Gamma(\beta(i+1)+1)} e^{-v j\left(\frac{\theta}{x}\right)^{k}} \\
& =v \beta k \theta^{k} x^{-k-1} \sum_{i=0}^{\infty} \frac{(-1)^{i}}{i !} \alpha^{i+1} \sum_{j=0}^{\infty} \frac{(-1)^{j} \Gamma(\beta(i+1)+1+j)}{j ! \Gamma(\beta(i+1)+1)}\left[e^{-v\left(\frac{\theta}{x}\right)^{k}}\right]^{\beta(i+1)} e^{-v j\left(\frac{\theta}{x}\right)^{k}} \\
& =v \beta k \theta^{k} x^{-k-1} \sum_{i=0}^{\infty} \sum_{j=0}^{\infty} \frac{(-1)^{i+j} \alpha^{i+1} \Gamma(\beta(i+1)+1+j)}{i ! j ! \Gamma(\beta(i+1)+1)}\left[e^{-v\left(\frac{\theta}{x}\right)^{k}}\right]^{\beta(i+1)+j} \\
& =v \beta k \theta^{k} x^{-k-1} e^{-b\left(\frac{\theta}{x}\right)^{k}} \sum_{i=0}^{\infty} \sum_{j=0}^{\infty} \frac{(-1)^{i+j} \alpha^{i+1} \Gamma(\beta(i+1)+1+j)}{i ! j ! \Gamma(\beta(i+1)+1)}\left[e^{-v\left(\frac{\theta}{x}\right)^{k}}\right]^{\beta(i+1)+j-1} \\
& =\alpha v \beta k \theta^{k} x^{-k-1} e^{-v\left(\frac{\theta}{x}\right)^{k}} \sum_{i=0}^{\infty} \sum_{j=0}^{\infty} \frac{(-1)^{i+j} \alpha^{i} \Gamma(\beta(i+1)+1+j)}{i ! j ! \Gamma(\beta(i+1)+1)}\left[e^{-v\left(\frac{\theta}{x}\right)^{k}}\right]^{\beta(i+1)+j-1} \\
& =g(x) \sum_{i=0}^{\infty} \sum_{j=0}^{\infty} \frac{(-1)^{i+j} \alpha^{i} \Gamma(\beta(i+1)+1+j)}{i ! j ! \Gamma(\beta(i+1)+1)}[G(x)]^{\beta(i+1)+j-1} \\
& =\sum_{i=0}^{\infty} \sum_{j=0}^{\infty} \frac{(-1)^{i+j} \alpha^{i}}{i ! j !} \frac{\Gamma(\beta(i+1)+1+j)}{\Gamma(\beta(i+1)+1)} g(x)[G(x)]^{\beta(i+1)+j-1} \\
& =\delta_{i, j} m g(x)[G(x)]^{m} \\
& \text { where } m=\beta(i+1)+j-1 \text { and } \delta_{i, j}=\sum_{i=0}^{\infty} \sum_{j=0}^{\infty} \frac{(-1)^{i+j}}{m i ! j !} \frac{\alpha^{i} \Gamma(\beta(i+1)+1+j)}{\Gamma(\beta(i+1)+1)}
\end{aligned}
$$

Equation (16) becomes the resulting equation from the above expansion. It is discovered from the resulting equation that the WEIW distribution can be articulated as a mixture version of the baseline distribution defined

\section{PROPERTIES OF THE WEIW DISTRIBUTION}

Some of the properties of the new distribution are conferred in this section.

\section{RELIABILITY AND HAZARD/FAILURE FUNCTIONS OF WEIW DISTRIBUTION}

The survival (reliability) function is derived and given as:

$$
R(x)=1-F(x)=\exp \left(-\alpha\left[\frac{\left(e^{-\left(\frac{\theta}{x}\right)^{k}}\right)^{v}}{1-\left(e^{-\left(\frac{\theta}{x}\right)^{k}}\right)^{v}}\right]^{\beta}\right)
$$

$x>0 ; \alpha, \beta>0$ and $v, k, \theta>0$

The hazard/failure rate function is obtained using:

$$
h(x)=\frac{f(x)}{1-F(x)}=\alpha \beta v k \theta^{k} x^{-k-1} \frac{\left[e^{-v\left(\frac{\theta}{x}\right)^{k}}\right]^{\beta}}{\left[1-e^{-v\left(\frac{\theta}{x}\right)^{k}}\right]^{\beta+1}}
$$

in equation (6). Also, some statistical properties of the WEIW can be derived from those of Exponentiated Inverse Weibull distribution.

$x>0 ; \alpha, \beta>0$ and $v, k, \theta>0$ 
The reversed hazard/failure rate function is obtained as:

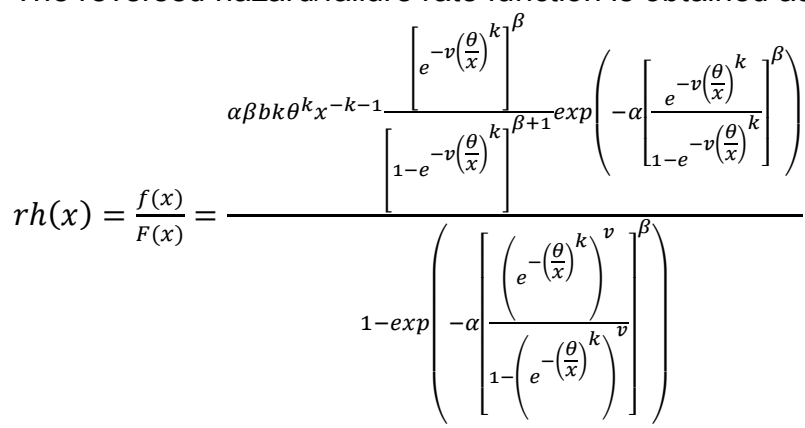

$x>0 ; \alpha, \beta>0$ and $, k, \theta>0$
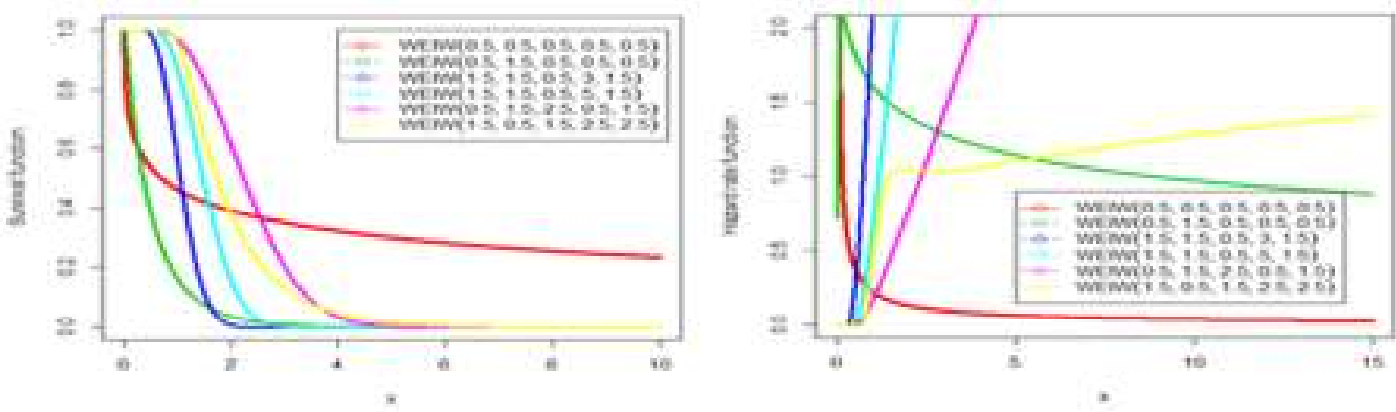

Figure 2: The Survival functions and Failure (Hazard) rate Plots

The failure/hazard rate function is described by different shapes such as decreasing, increasing, reversed J-shape and inverted bathtub depending on parameter values.

Theorem 1: If $X$ is a random variable from WEIW distribution, then the hazard rate function is of the form represented as:

$h_{W E I W}(x)=g_{E I W}(x) \frac{\left[G_{E I W}(x)\right]^{\beta-1}}{\left[R_{E I W}(x)\right]^{\beta+1}}$

Proof:

$h(x)=\alpha \beta v k \theta^{k} x^{-k-1} \frac{\left[e^{-v\left(\frac{\theta}{x}\right)^{k}}\right]^{\beta}}{\left[1-e^{-v\left(\frac{\theta}{x}\right)^{k}}\right]^{\beta+1}}=\alpha \beta v k \theta^{k} x^{-k-1} e^{-v\left(\frac{\theta}{x}\right)^{k}} \frac{\left[e^{-v\left(\frac{\theta}{x}\right)^{k}}\right]^{\beta-1}}{\left[1-e^{-v\left(\frac{\theta}{x}\right)^{k}}\right]^{\beta+1}}$

$=g(x) \frac{[G(x)]^{\beta-1}}{[1-G(x)]^{\beta+1}}=g(x) \frac{[G(x)]^{\beta-1}}{[R(x)]^{\beta+1}}$

where $\mathrm{R}(\mathrm{x})=1-\mathrm{G}(\mathrm{x}), x>0 ; \alpha, \beta>0$ and $, k, \theta>0$

\section{QUANTILE FUNCTION AND MEDIAN OF WEIW DISTRIBUTION}

Theorem 2: If $X$ is a random variable from WEIW distribution, then the quantile function is given as:

$Q(u)=\theta\left[-\log \left(\frac{\left[-\frac{1}{\alpha} \log (1-u)\right]^{1 / \beta}}{1+\left[-\frac{1}{\alpha} \log (1-u)\right]^{1 / \beta}}\right)^{1 / v}\right]^{-1 / k}$

The quantile function can be proved using the relation $Q(u)=F^{-1}(u)$, where $\mu$ is a random variable from uniform distribution on interval $(0,1)$

The median is obtained for the middle observation at $u=0.5$ and is given by;

$x($ median $)=\theta(22)$

Let $X$ be a random variable from the WEIW distribution, simulation can be done through the inverse transformation of a variable using uniform interval $\mu(0,1)$ and the random variable $X$ is taken as,

$X=\theta\left[-\log \left(\frac{\left[-\frac{1}{\alpha} \log (1-u)\right]^{1 / \beta}}{1+\left[-\frac{1}{\alpha} \log (1-u)\right]^{1 / \beta}}\right)^{1 / v}\right]^{-1 / k}$ 
MOMENT OF WEIW DISTRIBUTION

Moments are vital statistical measure for characterizing distributions; the $r^{\text {th }}$ moment for the WEIW distribution is derived as follows:

Theorem 3: Let $X$ be a random variable from the WEIW distribution, then the $r^{\text {th }}$ moment of $X$ is given by the following expression.

$w_{i, j} \theta^{r} v[\beta(i+1)+j]^{\frac{r}{k}} \Gamma\left(1-\frac{r}{k}\right)$

Proof:

From equation (14),

$$
\begin{gathered}
f(x)=\sum_{i=0}^{\infty} \sum_{j=0}^{\infty} \frac{(-1)^{i+j} \alpha^{i}}{i ! j !} \frac{\Gamma(\beta(i+1)+1+j)}{\Gamma(\beta(i+1)+1)} g(x)[G(x)]^{\beta(i+1)+j-1} \\
=\delta_{i, j} m g(x)[G(x)]^{m} \\
f(x)=\sum_{i=0}^{\infty} \sum_{j=0}^{\infty} \frac{(-1)^{i+j} \alpha^{i}}{i ! j !} \frac{\Gamma(\beta(i+1)+1+j)}{\Gamma(\beta(i+1)+1)} v k \theta^{k} x^{-(k+1)}\left[\left(e^{-\left(\frac{\theta}{x}\right)^{k}}\right)^{v}\right]^{\beta(i+1)+j} \\
f(x)=w_{i, j} v k \theta^{k} x^{-(k+1)}\left[\left(e^{-\left(\frac{\theta}{x}\right)^{k}}\right)^{v}\right]^{\beta(i+1)+j} \\
w_{i, j}=\sum_{i=0}^{\infty} \sum_{j=0}^{\infty} \frac{(-1)^{i+j} \alpha^{i}}{i ! j !} \frac{\Gamma(\beta(i+1)+1+j)}{\Gamma(\beta(i+1)+1)}
\end{gathered}
$$

The $r^{\text {th }}$ ordinary Moment of the WEIW distribution is given by:

$\mu_{r}^{\prime}=E(X)^{r}=\int_{0}^{\infty} x^{r} f(x, \square) d x$

$=w_{i, j} v k \theta^{k} \int_{0}^{\infty} x^{r-(k+1)} e^{-v[\beta(i+1)+j]\left(\frac{\theta}{x}\right)^{k}} d x$

THE MEAN AND VARIANCE OF WEIW DISTRIBUTION

$$
\begin{aligned}
& =w_{i, j} v[\beta(i+1)+j]^{\frac{r}{k}} \Gamma\left(1-\frac{r}{k}\right) \theta^{r} \\
& =w_{i, j} \theta^{r} v[\beta(i+1)+j]^{\frac{r}{k}} \Gamma\left(1-\frac{r}{k}\right)
\end{aligned}
$$

The mean of WEIW random variable $X$ is given by:

$E(X)=w_{i, j} \theta v[\beta(i+1)+j]^{\frac{1}{k}} \Gamma\left(1-\frac{1}{k}\right)$

Variance is obtained from $E\left(X^{2}\right)-[E(X)]^{2}$

Variance $=w_{i, j} \theta^{2} v[\beta(i+1)+j]^{\frac{2}{k}} \Gamma\left(1-\frac{2}{k}\right) \theta^{2}-\left\{w_{i, j} \theta v[\beta(i+1)+j]^{\frac{1}{k}} \Gamma\left(1-\frac{1}{k}\right)\right\}^{2}$

$w_{i, j} \theta v\left\{\theta[\beta(i+1)+j]^{\frac{2}{k}} \Gamma\left(1-\frac{2}{k}\right)-w_{i, j} \theta v\left\{[\beta(i+1)+j]^{\frac{1}{k}} \Gamma\left(1-\frac{1}{k}\right)\right\}^{2}\right\}$

$w_{i, j}=\sum_{i=0}^{\infty} \sum_{j=0}^{\infty} \frac{(-1)^{i+j} \alpha^{i}}{i ! j !} \frac{\Gamma(\beta(i+1)+1+j)}{\Gamma(\beta(i+1)+1)}$

\section{MOMENT GENERATING FUNCTION OF WEIW DISTRIBUTION}

The Moment generating function is derived as follows:

Theorem (4): If $X$ has WEIW distribution, then the moment generating function has the form expressed as;

$w_{i, j, r} \theta^{r} v[\beta(i+1)+j]^{\frac{r}{k}} \Gamma\left(1-\frac{r}{k}\right)$

Proof:

The Moment generating function for a continuous random variable is defined by:

$M_{X}(t)=E\left(e^{t X}\right)=\int_{0}^{\infty} e^{t X} f(x) d x$

$$
\begin{aligned}
& e^{t X}=\sum_{r=0}^{\infty}\left(1+\frac{t^{r} x^{r}}{r !}\right)=\sum_{r=0}^{\infty} \frac{t^{r} x^{r}}{r !}=\sum_{r=0}^{\infty} \frac{t^{r}}{r !} E\left(X^{r}\right) \\
& E\left(e^{t X}\right)=\sum_{r=0}^{\infty} \frac{t^{r}}{r !} w_{i, j} \theta^{r} v[\beta(i+1)+j]^{\frac{r}{k}} \Gamma\left(1-\frac{r}{k}\right)
\end{aligned}
$$

wherew $_{i, j}=\sum_{i=0}^{\infty} \sum_{j=0}^{\infty} \frac{(-1)^{i+j} \alpha^{i}}{i ! j !} \frac{\Gamma(\beta(i+1)+1+j)}{\Gamma(\beta(i+1)+1)}$

$E\left(e^{t X}\right)=w_{i, j, r} \theta^{r} v[\beta(i+1)+j]^{\frac{r}{k}} \Gamma\left(1-\frac{r}{k}\right)$

$w_{i, j, r}=\sum_{r=0}^{\infty} \sum_{i=0}^{\infty} \sum_{j=0}^{\infty} \frac{(-1)^{i+j} t^{r} \alpha^{i}}{i ! j ! r !} \frac{\Gamma(\beta(i+1)+1+j)}{\Gamma(\beta(i+1)+1)}$. 


\section{CHARACTERISTIC FUNCTION OF WEIW DISTRIBUTION}

The characteristics function for a continuous random variable $X$ fromWEIW distribution is given as follows $\emptyset_{X}(t)=E\left(e^{i t X}\right)=\int_{0}^{\infty} e^{i t x} f(x) d x$

$$
\begin{aligned}
& \int_{0}^{\infty}\left(1+i t x+\frac{(i t x)^{2}}{2 !}+\cdots\right) f(x) d x \\
=\sum_{r=0}^{\infty} \frac{(i t)^{r}}{r !} \int_{0}^{\infty} x^{r} f(x) d x=\sum_{r=0}^{\infty} \frac{(i t)^{r}}{r !} E(X)^{r} & \\
\emptyset_{X}(t) & =\sum_{r=0}^{\infty} \frac{(i t)^{r}}{r !} w_{i, j} \theta^{r} v[\beta(i+1)+j]^{\frac{r}{k}} \Gamma\left(1-\frac{r}{k}\right) \\
w_{i, j} & =\sum_{i=0}^{\infty} \sum_{j=0}^{\infty} \frac{(-1)^{i+j} \alpha^{i}}{i ! j !} \frac{\Gamma(\beta(i+1)+1+j)}{\Gamma(\beta(i+1)+1)}
\end{aligned}
$$

\section{DISTRIBUTION OF ORDER STATISTICS OF WEIW DISTRIBUTION}

Let $\mathrm{X}_{1}, \mathrm{X}_{2}, \ldots, \mathrm{X}_{\mathrm{n}}$ be independent and identically distributed random variables with associated order statistics $\mathrm{X}_{(1: \mathrm{n})}, \mathrm{X}_{(2: \mathrm{n})}, \ldots, \mathrm{X}_{(\mathrm{n}: \mathrm{n})}$ of size $\mathrm{n}$ from a distribution with density and cumulative functions denoted as $\mathrm{f}(\mathrm{x})$ and $\mathrm{F}(\mathrm{x})$ respectively, then the probability density function of the $\mathrm{r}^{\text {th }}$ order statistics $\mathrm{X}_{(\mathrm{r})}$ is given by:

$f_{X_{r: n}}(x)=\frac{1}{B(r, n-r+1)} F(x)^{r-1}(1-F(x))^{n-r} f(x)$

The $r^{\text {th }}$ order statistics for a WEIW random variable $X$ is derived using equation (12) and (13) in equation (31) as follows:

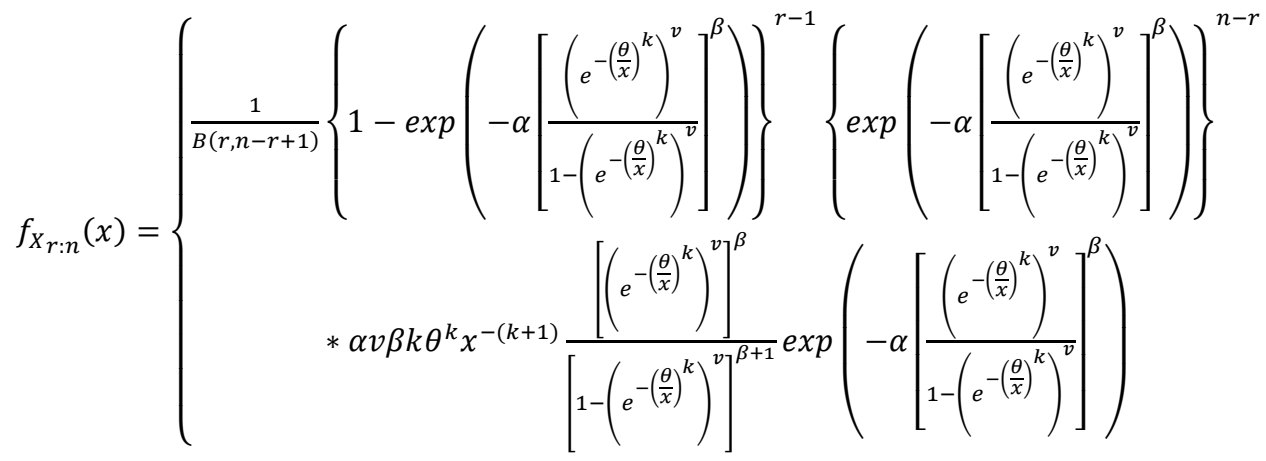

Using Binomial expansion, it can be re expressed as:

$f_{X_{r: n}}(x)=\frac{1}{B(r, n-r+1)} \sum_{i=0}^{n-r}(-1)^{r}\left(\begin{array}{c}n-r \\ i\end{array}\right) f(x)[F(x)]^{r+i-1}$

$f_{X_{r: n}}(x)=\left\{\begin{array}{c}\frac{1}{B(r, n-r+1)} \sum_{i=0}^{n-r}(-1)^{r}\left(\begin{array}{c}n-r \\ i\end{array}\right) \alpha v \beta k \theta^{k} x^{-(k+1)} \frac{\left[\left(e^{-\left(\frac{\theta}{x}\right)^{k}}\right)^{v}\right]^{\beta}}{\left[1-\left(e^{-\left(\frac{\theta}{x}\right)^{k}}\right)^{v}\right]^{\beta+1}} \\ * \exp \left(-\alpha\left[\frac{\left(e^{-\left(\frac{\theta}{x}\right)^{k}}\right)^{v}}{1-\left(e^{-\left(\frac{\theta}{x}\right)^{k}}\right)^{v}}\right]^{\beta}\right)\left\{1-\exp \left(-\alpha\left[\frac{\left(e^{-\left(\frac{\theta}{x}\right)^{k}}\right)^{v}}{\left.1-\left(e^{-\left(\frac{\theta}{x}\right)^{k}}\right)^{v}\right]}\right)\right\}^{r+i-1}\right.\end{array}\right.$

This minimum order statistics from equation (33) when $r=1$ is given by:

$$
f_{X_{1: n}}(x)=n \alpha v \beta k \theta^{k} x^{-(k+1)} \frac{\left[\left(e^{-\left(\frac{\theta}{x}\right)^{k}}\right)^{v}\right]^{\beta}}{\left[1-\left(e^{-\left(\frac{\theta}{x}\right)^{k}}\right)^{v}\right]_{*}^{\beta+1}}\left\{\exp \left(-\alpha\left[\frac{\left(e^{-\left(\frac{\theta}{x}\right)^{k}}\right)^{v}}{\left.1-\left(e^{-\left(\frac{\theta}{x}\right)^{k}}\right)^{v}\right]}\right)\right\}^{\beta}\right.
$$


This maximum order statistics from equation (26) when $r=n$ is given as:

$$
f_{X_{n: n}}(x)=\left\{\begin{array}{l}
\frac{1}{B(r, n-r+1)}\left\{1-\exp \left(-\alpha\left[\frac{\left(e^{-\left(\frac{\theta}{x}\right)^{k}}\right)^{v}}{\left.1-\left(e^{-\left(\frac{\theta}{x}\right)^{k}}\right)^{v}\right]}\right]^{\beta}\right\}\right\}^{n-1} \\
* \alpha v \beta k \theta^{k} x^{-(k+1)} \frac{\left[\left(e^{\left.-\left(\frac{\theta}{x}\right)^{k}\right)^{v}}\right]^{\beta}\right.}{\left[1-\left(e^{-\left(\frac{\theta}{x}\right)^{k}}\right)^{v}\right]^{\beta+1}} \exp \left(-\alpha\left[\frac{\left(e^{-\left(\frac{\theta}{x}\right)^{k}}\right)^{v}}{1-\left(e^{-\left(\frac{\theta}{x}\right)^{k}}\right)^{v}}\right]^{\beta}\right)
\end{array}\right.
$$

\section{(MAXIMUM LIKELIHOOD ESTIMATE (MLE)}

Let $X_{1}, X_{2}, \ldots, X_{n}$ be random sample of size $\mathrm{n}$ from the WEIW distribution, the method of maximum likelihood for estimating the unknown parameters is applied to the density function to obtain the likelihood and log-likelihood functions presented respectively as:

$L i k=\prod_{i=1}^{n}\left\{\alpha v \beta k \theta^{k} x^{-(k+1)} \frac{\left[\left(e^{-\left(\frac{\theta}{x}\right)^{k}}\right)^{v}\right]^{\beta}}{\left[1-\left(e^{-\left(\frac{\theta}{x}\right)^{k}}\right)^{v}\right]^{\beta+1}} \exp \left(-\alpha\left[\frac{\left(e^{\left.-\left(\frac{\theta}{x}\right)^{k}\right)^{v}}\right.}{1-\left(e^{-\left(\frac{\theta}{x}\right)^{k}}\right)^{v}}\right]\right\}\right.$

$\log L i k=\left\{\begin{aligned} n \log (\alpha)+n \log (v)+n \log (\beta)+n \log (k)+n k \log (\theta)-(k+1) \sum_{i=1}^{n} x-v \beta \theta^{k} \sum_{i=1}^{n} \frac{1}{x} \\ -(\beta+1) \sum_{i=1}^{n} \log \left[1-\left(e^{-\left(\frac{\theta}{x}\right)^{k}}\right)^{v}\right]+\sum_{i=1}^{n}\left(-\alpha\left[\frac{\left(e^{-\left(\frac{\theta}{x}\right)^{k}}\right)^{v}}{1-\left(e^{-\left(\frac{\theta}{x}\right)^{k}}\right)^{v}}\right]^{\beta}\right)\end{aligned}\right.$

$\frac{d L L}{d \alpha}=\frac{n}{\alpha}-\sum_{i=1}^{n}\left(\left[\frac{\left(e^{-\left(\frac{\theta}{x}\right)^{k}}\right)^{v}}{1-\left(e^{-\left(\frac{\theta}{x}\right)^{k}}\right)^{v}}\right]^{\beta}\right)$

$\frac{d L L}{d \beta}=\frac{n}{\beta}-v \theta^{k} \sum_{i=1}^{n} \frac{1}{x}-\sum_{i=1}^{n} \log \left[1-\left(e^{-\left(\frac{\theta}{x}\right)^{k}}\right)^{v}\right]-\alpha \sum_{i=1}^{n}\left[\frac{\left(e^{-\left(\frac{\theta}{x}\right)^{k}}\right)^{v}}{1-\left(e^{-\left(\frac{\theta}{x}\right)^{k}}\right)^{v}}\right]^{\beta} \log \frac{\left(e^{-\left(\frac{\theta}{x}\right)^{k}}\right)^{v}}{1-\left(e^{-\left(\frac{\theta}{x}\right)^{k}}\right)^{v}}$

$\left\{\begin{array}{l}\frac{d L L}{d k}=\frac{n}{k}+n \log (\theta)-\sum_{i=1}^{n} x-v \beta \theta^{k} \log (\theta) \sum_{i=1}^{n} \frac{1}{x}-(\beta+1) \sum_{i=1}^{n} \frac{e^{-v\left(\frac{\theta}{x}\right)^{k}\left(\frac{\theta}{x}\right)^{k} \log \left(\frac{\theta}{x}\right)}}{\left[1-\left(e^{-\left(\frac{\theta}{x}\right)^{k}}\right)^{v}\right]} \\ -\alpha \beta\left[1-\left(e^{-\left(\frac{\theta}{x}\right)^{k}}\right)^{v}\right]^{\beta}\left[e^{-v\left(\frac{\theta}{x}\right)^{k}}\right]^{\beta}\left(\frac{\theta}{x}\right)^{k} \log \left(\frac{\theta}{x}\right)\left\{1+\left[e^{-v\left(\frac{\theta}{x}\right)^{k}}\right]^{\beta}\left[1-\left(e^{\left.-\left(\frac{\theta}{x}\right)^{k}\right)^{v}}\right]^{-1}\right\}\right.\end{array}\right.$

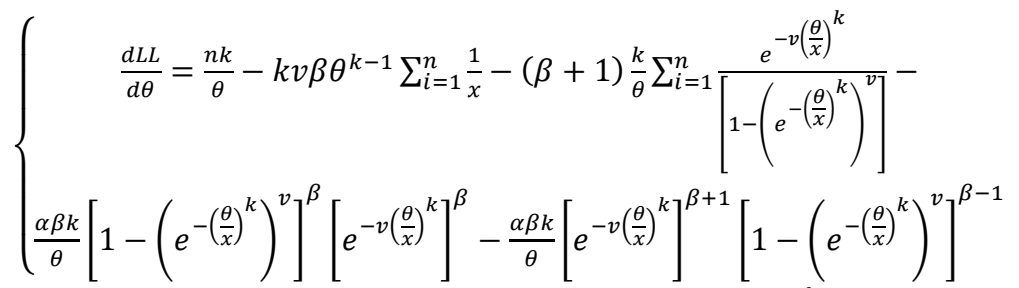

$\left\{\begin{array}{c}\frac{d L L}{d v}=\frac{n k}{\theta}-\beta \theta^{k} \sum_{i=1}^{n} \frac{1}{x}-(\beta+1) \frac{k}{\theta} \sum_{i=1}^{n} \frac{e^{-v\left(\frac{\theta}{x}\right)^{k}\left(\frac{\theta}{x}\right)^{k}}\left[1-\left(e^{-\left(\frac{\theta}{x}\right)^{k}}\right)^{v}\right]}{\left.\left[e^{-\left(\frac{\theta}{x}\right)^{k}}\right)^{v}\right]^{\beta}\left[e^{-v\left(\frac{\theta}{x}\right)^{k}}\right]^{\beta}\left(\frac{\theta}{x}\right)^{k}+\beta\left[e^{-v\left(\frac{\theta}{x}\right)^{k}}\right]^{\beta+1}\left[1-\left(e^{-\left(\frac{\theta}{x}\right)^{k}}\right)^{v}\right]^{\beta-1}\left(\frac{\theta}{x}\right)^{k}} \\ \beta[1-\alpha\end{array}\right.$ 
The derivatives of the log-likelihood function with respect to the unknown parameters when equated to zero for simultaneous solutions can provide us with the estimates which is analytically difficult owing to the complex nature of the function, hence the Newton Raphson Algorithm in R-software could be employed for obtaining numerical solutions for the estimates. The confidence intervals are obtainable from the inverse dispersion matrix $I^{-1}(\widehat{\varnothing})$ which can be generated from second derivatives of log Likelihood function.

The $100(1-p) \%$ two sided confidence intervals for the parameters are of the form:

$\hat{\beta} \pm Z_{p / 2} \sqrt{I^{-1}{ }_{\beta \beta}(\widehat{\varnothing})}, \hat{k} \pm Z_{p / 2} \sqrt{I^{-1}{ }_{k k}(\widehat{\varnothing})} ; \quad \hat{b} \pm Z_{p / 2} \sqrt{I^{-1}{ }_{b b}(\widehat{\varnothing})} ;$

$\hat{\alpha} \pm Z_{p / 2} \sqrt{I^{-1}{ }_{\alpha \alpha}(\widehat{\varnothing})}$ and $\hat{\theta} \pm Z_{p / 2} \sqrt{I^{-1}{ }_{\theta \theta}(\widehat{\varnothing})}$

where $\square_{\square}$ is used as the $100(1-p) \%$ upper percentile of the standard normal distribution.

\section{DISCUSSION OF RESULTS I APPLICATION}

The findings from the study and application to real life examples are presented in this section.

\section{APPLICATION TO REAL DATASET}

In this section, we demonstrate the superiority of the proposed distribution WEIW using real data sets in reliability engineering to select the best model among the competing models. A statistical software (R-4.0.3) is used to obtain the MLE of the model parameters. For the model selection, the criteria used are Akaike information criterion (AIC) and Bayesian information criterion (BIC) as:

$A I C=2 k-2 \ln (L)$ and $B I C=2 \ln (L)+k \ln (n)$ where $\mathrm{k}$ is the number of parameters in the model and $L$ is the maximized value of the likelihood function for the model. The AIC is the measure of the relative quality of a statistical model for a given set of data. BIC is also a criterion for model selection among a finite set

of models and is closely related to the AIC. In Table 2, the IEW model is more appropriate in terms of $\mathrm{AIC}$ and $\mathrm{BIC}$ since the values of $\mathrm{AIC}$ and BIC of the WEIW model were smallest among the competing models. Therefore, we can conclude that the performance of the WEIW model is better.

The output values from goodness of fit for making decisions were also generated for $L L$ P-values and Kolgomorv Smirnoff (K-S) statistics which are also choice of better model selection criteria. The model with best fit to the data is expected to have the smallest estimated value of estimated model criteria.

The dataset on tests of the endurance of deep groove ball bearings from [28] was adopted and applied to the WEIW distribution and other competing family-related models. The results are presented in Table 1 and Figure
3.

Table 1: Maximum Likelihood Estimates and Criteria for Model Selection for drug-resistant tuberculosis on Ball Bearings Data

\begin{tabular}{|l|l|l|l|l|l|l|}
\hline Models & Parameter Estimates & AIC & BIC & LL & K-S & $\begin{array}{l}\text { P- } \\
\text { value }\end{array}$ \\
\hline WEIW & $\hat{\alpha}=0.6712, \hat{\beta}=0.0771, \hat{\theta}=16.1518, \hat{b}=3.0251, \hat{k}=0.9797$ & 778.430 & 789.741 & 384.377 & 0.1343 & 0.1344 \\
\hline IW & $\hat{\theta}=53.0868, \hat{k}=1.4208$ & 779.600 & 784.123 & 387.798 & 0.1617 & 0.1618 \\
\hline EIW & $\hat{\theta}=53.4332, \hat{b}=1.0032, \hat{k}=1.7858$ & 797.072 & 803.858 & 395.534 & 0.1672 & 0.1673 \\
\hline St.EIW & $, \hat{b}=12.9671, \hat{k}=0.6857$ & 835.268 & 839.791 & 415.632 & 0.2313 & 0.2314 \\
\hline
\end{tabular}
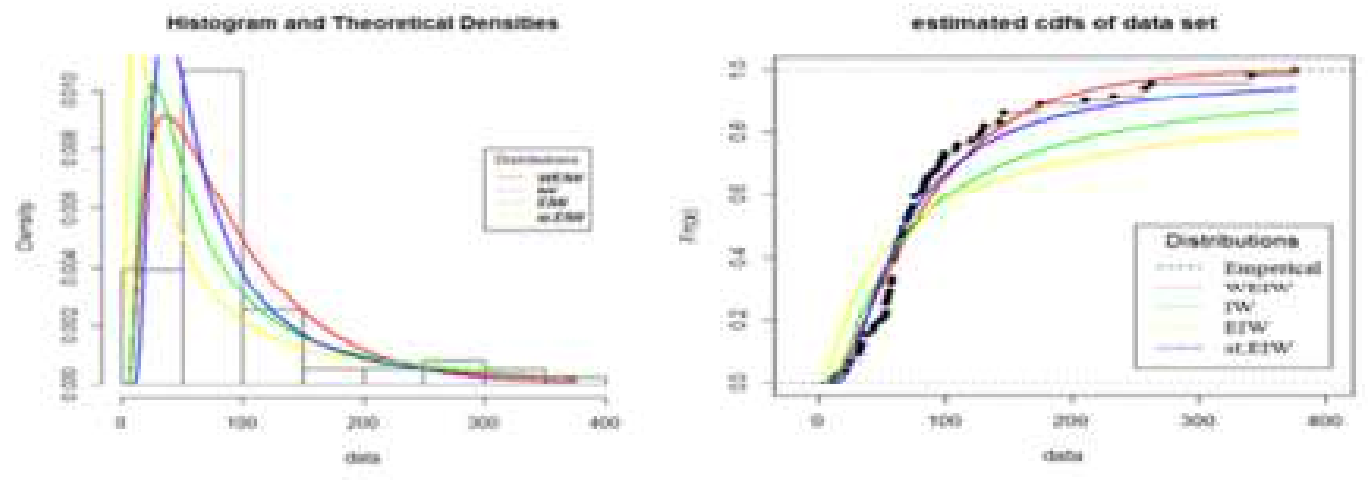

Figure 3: Plots of the pdf and cdf of distributions on Ball Bearings Data From Table 1, it is revealed that the WEIW has the smallest values of chosen model criteria and the smallest Kolmogorov Smirnoff test statistics; it has also revealed that the WEIW has the largest $p$-value and the lowest values 
three-parameter EIW and the standard two-parameter st.EIW gave a worse fit than IW. Figure 3 which characterize the density and estimated cdf plots with the other related models. Figure 3 which characterizes the density and estimated cdf plots with the other related models, also reinforced the conclusions from the computations in Table 3 that WEIW is the best choice for modeling these dataset.

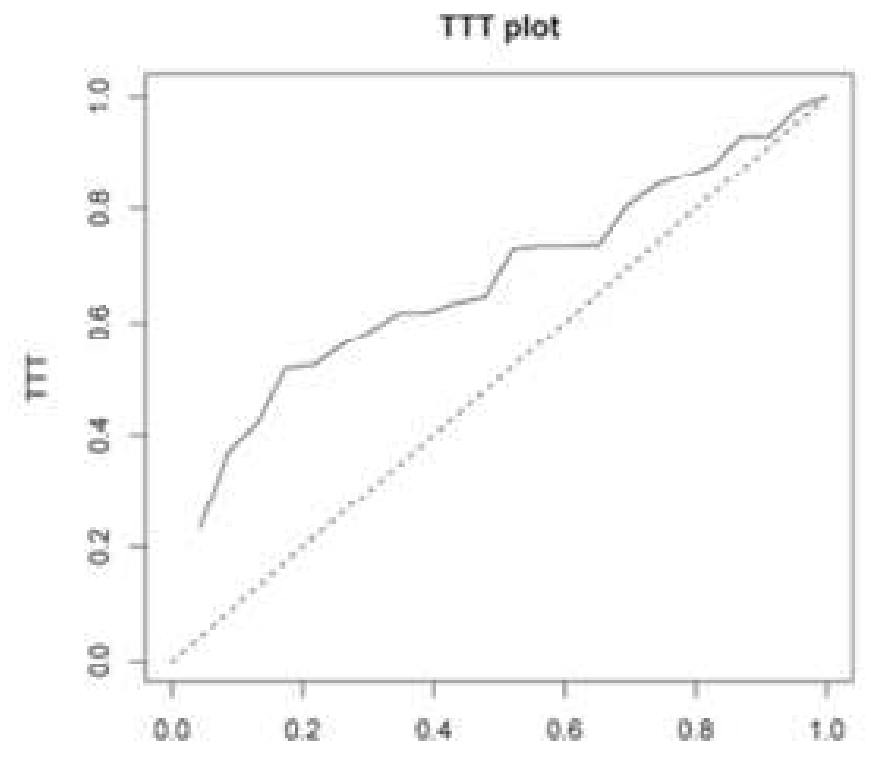

Figure 4:The TTT plot for the endurance of deep groove ball bearings data. TTT $=$ total time on test.

Figure 4 provides a total time on test (TTT) plot for the durability of deep groove ball bearings data. Since the

\section{CONCLUSIONS}

The Exponentiated Inverted Weibull (EIW) model has been extended to obtain the WeibullExponentiated Inverted Weibull distribution (WEIW). The WEIW addresses some of the limitations identified with the EIW and also provides better flexibility than the EIW and the IW models. The statistical properties studied show that the distribution is positively skewed; the shape can be unimodal, approximately symmetric and is suitable for modeling right-shaped (positively skewed) data. The parameters of distribution were estimated using maximum likelihood estimation. A real-life dataset was used to examine its performance, and results from data analysis revealed that the WEIW distribution has the capacity to provide a better fit for modeling the real-life data.

\section{AUTHORS' CONTRIBUTIONS}

All the authors contributed significantly in writing this article. The authors read and approved the final manuscript.

\section{REFERENCES}

Alzaatreh A, Lee C and Famoye F. A new method for generating families of continuous distributions, Metron 2013; 71: 63-79.

Bourguignon M, Silva RB and Cordeiro GM. The Weibull-G Family of Probability Distributions. Journal of Data Science 2014; 12: 53-68.

Alzaatreh A, Famoye F and Lee C. Weibull-Pareto plot is concave and lying above the line, it means that its distribution may have an increasing hazard rate. Therefore it can be properly accommodated by a WEIW $(\alpha, \beta, \lambda)$ model with increasing failure rate.

in Statistics-Theory and Methods, 42, 2013; 1673-1691.

Aljarrah MA, Famoye $F$ and Lee C.A new WeibullPareto distribution. Communication in Statistics Theory and methods 2015; 44(19): 4077- 4095.

Merovci F and Elbatal I. Weibull Rayleigh Distribution: Theory and Applications. Applied Mathematics and Information Sciences 2015; 5: 1-11.

Oguntunde PE, Balogun OS, Okagbue $\mathrm{HI}$ and Bishop SA. The Weibull Exponential Distribution: Its Properties and Applications. Journal of Applied Sciences2015; 15: 1305-1311.

Yahaya A and Sa'ad Mohammed. On Weibull-Burr XII Distribution: Properties and Applications. Nigerian Journal of Technological Research 2019; 14(1): DOI:10.4314/nijtr.v14i1.6

Voda VG. On the inverse Rayleigh distributed random variable, Rep. Statist. Appl. Res. Un. Japan Science Engrs1972; 19: 13-21.

Drapella A. Complementary Weibull Distribution: Unknown or Just Forgotten. Quality and Reliability Engineering International 1993; 9: 383- 385.

Mudholkar GS, and Kolia, GD. Generalized Weibull Family: A structural Analysis. Communications in Statistics Theory and Methods 1994; 23(4): 1149-1171. 
Khan MS, Pasha GR and Pasha AH. Theoretical Analysis of Inverse Weibull distribution. WSEAS Tran. Mathematics 2008; 7(8): 30-38.1.

De GusmaoFRS, Ortega EMM and Cordeiro GM.The generalized inverse Weibull distribution. Statistical Papers 2011; DOI 10.1007/s00362009-0271-3.

Elbatal I and Muhammed HZ.Exponentiated Generalized Inverse Weibull Distribution. Applied Mathematical Sciences 2014; 8(81): 3997-4012.

Fatima K, and Ahmad SP. Weighted inverse Rayleigh distribution. International Journal of Statistics and Systems 2017; 12(1): 119-137.

Oguntunde PE, Adejumo AO and Owoloko EA. The Weibull-Inverted Exponential Distribution: Ageneralization of the Inverse Exponentail Distribution. In World Congress on Engineering, July 5-7, 2017; London U.K.

Fayomi A. The Odd Frechet inverse Weibull Distribution with application. Journal of Nonlinear Sciences and Applications 2019; 12: 165-172.

Elgarhy $M$ and Alrajhi S. The odd Frechet Inverse Rayleigh distribution: Statistical Properties and Applications, Journal of Nonlinear Sci. 2019; 12: 291-299.DOI:10.22436/jnsa.012.05.03.

Aldahlan MA. The Inverse Weibull Inverse Exponential Distribution with Applications. International Journal of Contemporary Mathematical Sciences 2019; 14(1): 17-30. https://doi.org/10.12988/ijcms.2019.913.

Almarashi AM, Badr MM, Elgarhy $M$, Jamal $F$ and Chesneau C. Statistical Inference of the HalfLogistics Inverse Rayleigh Distribution. Article in entropy 2020; DOI:10..3390/e22040449.

Flaih A, Elssalloukh $\mathrm{H}$, Mendi $\mathrm{E}$ and Milanova $\mathrm{M}$. The Exponentiated Inverted Weibull Distribution, Appl. Maths. Inf. Sciences 2012; 6(2): 161-171.

Aljuaid A. Estimating the Parameters of Exponentiated Inverted Weibull Distribution under Type-II Censoring. Appl.Math. Sciences 2013; 7(35): 1721-1736.

Hassan AS, Marwa AA, Zaher A and Elsherpieny EA. Comparison of Estimators for Exponentiated Inverted Weibull Distribution based on Grouped Data. Int. Journal of Engineering and Application 2014; 4(4): 77-90

Ramos PL and Loucada F. Comments on the Exponentiated Inverted Weibull Distribution. Appl. Math. Inf. Sciences2016; 10(5): 16411943.

Rao GS and Mbwambo S.Exponentiated Inverse Rayleigh Distribution and an Application to Coating Weights of Iron Sheets Data. Journal of
Probability and Statistics 2019; 20(19): Article

ID:

7519429

https:doi.org/10.1155/2019/7519492.

Jan U, Fatima $\mathrm{K}$ and Ahmad SP. Transmuted Exponentiated Inverse Weibull Distribution with application in Medical Sciences. International Journal of Mathematics Trends and Technology (IJMTT) 2017; 50(3): 160-167.

Kelle AZ, Kamath ARR and Perera UD. Reliability Analysis of NCN machine tools. Reliability Engineering 1982; 3: 449-473.

Bjerkedal T. Acquisition of resistance in guinea pigs infected with different doses of virulent tubercle bacilli, American Journal of Epidemiology 1960; 72(1): 130-140.

Lawless, J. F (1982).Statistical Models and Methods for Lifetime Data. New York: Wiley. 
